\title{
MACROECONOMIA
}

\section{Uma estimativa do produto potencial no $\mathrm{Brasil}^{\dagger}$}

Fernando de Holanda Barbosa Filho*

RESUMO - Este artigo calcula o produto potencial da economia brasileira com dados de 1995 a 2010. O artigo mostra que a taxa de crescimento do produto potencial atingiu um pico de $4,4 \%$ no período 2007-2010 e que a taxa de crescimento do mesmo deve cair nos próximos anos por dois motivos: a contribuição do aumento das horas trabalhadas deve diminuir nos próximos anos, reduzindo a contribuição das mesmas no crescimento do produto potencial, e a taxa de crescimento da PTF no Brasil deve crescer somente 0,6\% nos anos de 2011 e 2012 caso as expectativas de crescimento da economia se confirmem. Estes dois fatos indicam que o crescimento do produto potencial da economia deve se encontrar abaixo dos $4 \%$ ao ano nos próximos anos.

Palavras-chave: Crescimento econômico. PIB potencial.

\section{INTRODUÇÃO}

A taxa de crescimento do produto potencial brasileiro é uma informação importante para a condução de política econômica, pois permite à autoridade monetária saber o quanto o país pode crescer de modo consistente com a meta de inflação.

Este artigo calcula a taxa de crescimento do produto potencial no Brasil nos últimos anos e projeta esta taxa para os próximos anos. Para atingir este objetivo, o artigo utiliza a evolução dos fatores de produção (capital, trabalho e capital humano) e projeta cenários para calcular a taxa de crescimento futuro do produto potencial da economia brasileira.

Os cálculos realizados mostram que a taxa de crescimento do produto potencial brasileiro nos últimos anos ficou em torno dos 4,4\% ao ano, um número próximo ao estimado pela previsão, que gira entre $4 \%$ e $4,5 \%$ ao ano.

$\mathrm{O}$ artigo argumenta ainda que o produto potencial brasileiro deve cair $0,3 \%$ devido à redução da elevação do total de horas trabalhadas, visto que não parece haver mais espaços para redução da taxa de desemprego. Adicionalmente, o artigo mostra que a taxa de crescimento do produto potencial da economia brasileira esteja mais próxima dos 3,5\% do que dos 4,5\% nos

\footnotetext{
$\bar{\dagger}$ Trabalho realizado antes da atualização dos dados do IBGE de dezembro de 2011. O autor agradece ao INCT $(\mathrm{CNPq})$ e FAPERJ pelo auxílio financeiro.

* Doutor em Economia pela Universidade de Nova York. É pesquisador do Centro de Desenvolvimento Econômico do Instituto Brasileiro de Economia e professor da Fundação Getúlio Vargas. Endereço eletrônico: fernando. filho@fgv.br.
} 
próximos anos, devido a uma queda na PTF de 2011.

O artigo está organizado em cinco seções, incluindo esta introdução. A segunda seção apresenta a metodologia utilizada para calcular o produto potencial da economia brasileira. A terceira seção apresenta os resultados da produtividade total dos fatores. A quarta seção realiza o cálculo do produto potencial com base nas taxas de crescimento observadas nos dados. A quinta seção projeta a taxa de crescimento do produto potencial no futuro e a sexta seção conclui o trabalho.

\section{METODOLOGIA}

A taxa de crescimento do produto potencial da economia brasileira será calculada com base na metodologia da função de produção, admitindo uma função Cobb-Douglas. O crescimento dos fatores de produção capital $(K)$, trabalho $(L)$, capital humano $(H)$ e o crescimento da produtividade total dos fatores $(A)$ são os insumos necessários para o cálculo do produto potencial.

A taxa de crescimento do total de horas trabalhadas e do estoque de capital físico são obtidas dos dados. A variação do estoque de capital humano e da PTF são obtidos de Barbosa Filho (2011). Com base na taxa de crescimento da PTF (calculada), obtêm-se a taxa de crescimento do produto potencial para diversos períodos baseando-se na variação dos fatores, da PTF e da hipótese adicional de plena utilização da capacidade instalada.

\subsection{FUNÇÃO DE PRODUÇÃO}

A função de produção é dada por $Y_{t}=A_{t} F\left(u_{t} K_{t}, H_{t} L_{t}\right)$, em que $Y_{t}$ é o produto agregado, $A_{t}$ é a produtividade total dos fatores (PTF), $u_{t}$ é o índice de utilização da capacidade instalada (NUCI), $K_{t}$ é o estoque de capital físico disponível na economia, $u_{t} K_{t}$ é o total de serviços produtivos do capital utilizado na produção, $H_{t}$ é o capital humano por trabalhador e $L_{t}$ é o número de horas trabalhadas.

A função de produção é dada pela especificação Cobb-Douglas:

$$
Y_{t}=A_{t}\left(u_{t} K_{t}\right)^{\alpha}\left(H_{t} L_{t}\right)^{1-\alpha}
$$

em que $\alpha$, a elasticidade do produto em relação ao capital, é igual à participação do capital na renda em equilíbrio competitivo. Admite-se que $\alpha=0,4$, com base em Gomes, Pessôa e Veloso (2003). 


\subsection{TOTAL DE HORAS TRABALHADAS}

O total das horas trabalhadas foi obtido com base na PNAD e em Barbosa Filho e Pessôa (2009). Neste trabalho, os autores constroem uma série de horas trabalhadas utilizando os dados de horas totais trabalhadas da PNAD e utilizando os dados da PME para adicionar a variação mensal na série. Logo, na análise da decomposição do PIB para se computar a PTF utiliza-se a média de horas trabalhadas mensais para cada ano.

\subsection{ESTOQUE DE CAPITAL FÍSICO}

O estoque de capital físico é calculado com base no método do inventário perpétuo:

$$
K_{t+1}=(1-\delta) K_{t}+I_{t}
$$

em que $K_{t}$ é o nível de capital inicial, $K_{t+1}$ é o nível de capital do período seguinte, $\delta$ é a taxa de depreciação e $I_{t}$ é o investimento.

Para obter uma série de capital é necessário que se possua um nível de capital inicial, uma taxa de depreciação e o investimento da economia para cada período. Para calcularmos o estoque de capital inicial, assumimos que a relação capital-produto em 1970 era de 2,36, com base em Gomes, Pessôa e Veloso (2003). A taxa de depreciação do capital físico empregada nesse estudo é igual a 3,5\%. Os dados de investimento foram obtidos da série de Formação Bruta de Capital Fixo a preços constantes do Sistema de Contas Nacionais (SCN) do IBGE. O estoque de capital foi ajustado pelo grau de utilização da capacidade, $u_{t}$, da Fundação Getúlio $\operatorname{Vargas}(\mathrm{FGV})$.

\subsection{CÁLCULO DO CAPITAL HUMANO}

A mensuração do capital humano é um tema bastante controverso no meio acadêmico. Entretanto, após estudos iniciais como Mankiw, Romer e Weil (1992) que utilizam taxas de matrícula como forma de mensurar o estoque de capital humano, a literatura econômica passou a utilizar os dados de anos médios de escolaridade da população economicamente ativa (PEA). Neste trabalho, utilizamos as estimativas de capital humano de Barbosa Filho (2011), onde o capital humano é medido com base em duas metodologias distintas: Bils e Klenow (2000, daqui em diante BK) e Barbosa Filho, Pessôa e Veloso (2010, daqui em diante BPV).

A Tabela 1 mostra a diferença entre as duas medidas de capital humano. Com base nos anos médios de escolaridade, fruto do processo de universalização da educação, o estoque de capital humano à la BK se eleva todo ano enquanto que o proposto por BPV possui maior 
variabilidade em decorrência de se levar em conta a variação do retorno da educação em todos os períodos, diferentemente de BK, que supõe o mesmo retorno da educação para diferentes $\operatorname{anos}^{1}$.

TABELA 1 - ESTOQUE DE CAPITAL HUMANO

\begin{tabular}{c|rrr}
\hline Ano & Anos de escolaridade (h) & \multicolumn{1}{c|}{ BK } & BPV \\
\hline 1995 & 5,5 & 4,8 & 3,3 \\
1996 & 5,7 & 4,9 & 3,3 \\
1997 & 5,8 & 4,9 & 3,5 \\
1998 & 6,0 & 5,0 & 3,6 \\
1999 & 6,1 & 5,1 & 3,6 \\
2000 & 6,3 & 5,2 & 3,6 \\
2001 & 6,4 & 5,3 & 3,5 \\
2002 & 6,6 & 5,4 & 3,6 \\
2003 & 6,8 & 5,5 & 3,7 \\
2004 & 6,9 & 5,6 & 3,7 \\
2005 & 7,0 & 5,6 & 3,8 \\
2006 & 7,2 & 5,7 & 3,7 \\
2007 & 7,3 & 5,8 & 3,9 \\
2008 & 7,5 & 5,9 & 3,9 \\
2009 & 7,6 & 6,0 & 3,8 \\
\hline
\end{tabular}

FONTE: Barbosa Filho (2011).

\subsection{CÁLCULO DA PTF}

A produtividade total dos fatores da economia brasileira é uma variável crucial para o cálculo do produto potencial. Uma elevada variação da PTF possibilita um produto potencial maior e uma PTF baixa reduz o mesmo. Com base em (1), a PTF pode ser obtida da seguinte fórmula:

$$
A_{t}=\frac{Y_{t}}{\left(u K_{t}\right)^{\alpha}\left(L_{t} H_{t}\right)^{1-\alpha}}
$$

\subsection{CÁLCULO DO PRODUTO POTENCIAL}

Obtida a taxa de crescimento da PTF pode-se calcular a taxa de crescimento do produto potencial $\left(\frac{\dot{Y}}{Y}\right)$ conforme a Equação (4):

$$
\frac{\dot{Y}}{Y}=\frac{\dot{A}}{A}+\alpha \frac{\dot{K}}{K}+(1-\alpha)\left(\frac{\dot{L}}{L}+\frac{\dot{H}}{H}\right)
$$

onde $\frac{\dot{A}}{A}$ é a taxa de crescimento da PTF, $\frac{\dot{K}}{K}$ é a taxa de crescimento do estoque de capital, $\frac{\dot{L}}{L}$ é a taxa de crescimento das horas trabalhadas e $\frac{\dot{H}}{H}$ é a taxa de crescimento do capital humano. A Equação (4) é obtida através da diferenciação no tempo da função de produção (1) e da hipótese de que a economia encontra-se em pleno emprego, a capacidade instalada não

1 Para mais detalhes ver Barbosa Filho (2011). 
varia: $\frac{\dot{u}}{u}=0$.

\section{PRODUTIVIDADE TOTAL DOS FATORES}

A PTF utilizada neste trabalho é obtida de Barbosa Filho (2011). O autor calcula a PTF utilizando duas mensurações de capital humano: à la Bils e Klenow (BK) e à la Barbosa Filho, Pessôa e Veloso (BPV). Para o cálculo do estoque de capital humano estimado para os anos de 2010 e 2011, o autor estima um crescimento baseado no crescimento do estoque de anos de escolaridade para o à la $\mathrm{BK}$ e um crescimento de $1 \%$ no estoque de capital humano mensurado à la BPV.

\subsection{RESULTADOS}

Como dito anteriormente, a PTF foi calculada com base em duas séries de capital humano distintas, uma à la Bils e Klenow e uma à la Barbosa Filho, Pessôa e Veloso. A Tabela 2, abaixo, mostra a PTF calculada para diversos períodos conforme as duas metodologias em diversos subperíodos entre os anos de 1995 e 2009.

TABELA 2 - PTF POR TIPO DE CAPITAL HUMANO

FONTE: Barbosa Filho (2011).

\begin{tabular}{l|r|r}
\hline \multirow{2}{*}{ Período } & \multicolumn{2}{|c}{ Variação } \\
\cline { 2 - 3 } & \multicolumn{1}{c}{ BK } & \multicolumn{1}{c}{ BPV } \\
\hline 2011 (estimativa) & $0,6 \%$ & $0,6 \%$ \\
$1995-2010$ & $-0,1 \%$ & $0,2 \%$ \\
$2003-2010$ & $0,5 \%$ & $0,8 \%$ \\
$2007-2010$ & $1,3 \%$ & $1,5 \%$ \\
$2003-2008$ & $0,4 \%$ & $0,6 \%$ \\
$2004-2008$ & $1,1 \%$ & $1,4 \%$ \\
\hline
\end{tabular}

\subsection{ANÁLISE DOS RESULTADOS}

As duas medidas de PTF mostram pouca variação em relação ao ano inicial de 1995, com um crescimento anual de apenas $0,2 \%$ ao ano, à la BPV. Assim como em Barbosa Filho, Pessôa e Veloso (2010), observa-se uma queda da PTF até o ano de 2004 e depois uma recuperação da mesma.

Nos anos de 2004 a 2008 o crescimento da PTF acelera, com a mesma crescendo 1,1\% com capital humano de BK e 1,4\% com capital humano de BPV. O período de maior elevação da PTF em ambos as medidas ocorre no período 2007-2010, onde ela atinge 1,3\% e $1,5 \%$, respectivamente.

A PTF é mais volátil na medida BPV do que na medida de BK porque com os ciclos econômicos a produtividade do capital humano também varia, o que ocasiona oscilações na 
medida $\mathrm{BPV}^{2}$.

Por último, observa-se nas Tabela 2 uma redução da PTF entre 2008 e 2011, o que pode reduzir a taxa de crescimento do produto potencial da economia.

\section{CÁLCULO DO PRODUTO POTENCIAL}

A taxa de crescimento do produto potencial é afetada pela taxa de crescimento do estoque de capital ${ }^{3}$, pela taxa de crescimento das horas trabalhadas, pela taxa de crescimento do capital humano e pela taxa de crescimento da PTF. Nesta seção será calculada a taxa de crescimento do produto potencial utilizando a função de produção representada pela Equação (1).

\subsection{TAXA DE CRESCIMENTO DO CAPITAL}

A taxa de crescimento do capital é dada pela Equação (5):

$$
\frac{\dot{K}}{K}=\frac{(I / Y)}{(K / Y)}-\delta
$$

O estoque de capital se eleva quando a taxa de investimento dividida pela relação capital produto é maior do que a taxa de depreciação. Desta forma, a taxa de crescimento do estoque de capital depende fundamentalmente da taxa de investimento da economia brasileira.

Com base nos dados de PIB, formação bruta do capital fixo e deflatores do PIB (obtidos do IBGE), pode-se calcular a taxa de investimento em valores constantes. Esta atingiu o seu pico em 2010, com 18,4\%. Entretanto, na média do período a taxa foi extremamente baixa, com um investimento médio superior aos 17\% do PIB somente no período 2007-2010. A Tabela 3, abaixo, mostra a taxa de investimento da economia brasileira em valores constantes de 2010.

TABELA 3 - TAXA DE INVESTIMENTO E DE CRESCIMENTO DO CAPITAL (MÉDIA POR PERÍODO)

\begin{tabular}{c|r|r}
\hline Período & Taxa de investimento & Crescimento do capital \\
\hline $1995-2010$ & $16,4 \%$ & $2,5 \%$ \\
$2003-2010$ & $16,1 \%$ & $2,5 \%$ \\
$2007-2010$ & $17,4 \%$ & $3,2 \%$ \\
$2003-2008$ & $15,6 \%$ & $2,3 \%$ \\
$2004-2008$ & $15,9 \%$ & $2,5 \%$ \\
\hline
\end{tabular}

FONTE: Elaboração própria.

A Tabela 3 mostra que a maior taxa de investimento médio foi de 17,4\% entre 2007 e 2010, quando a variação de capital atingiu 3,2\% ao ano. Entretanto, a taxa de investimento 2 Para mais detalhes acerca da volatilidade de BPV, ver Barbosa Filho, Pessôa e Veloso (2010).

$3 \mathrm{O}$ nível de utilização da capacidade instalada se mantém constante no cálculo do produto potencial. A variação do NUCI ocorre durante os ciclos econômicos. O NUCI baixa nas recessões, o que possibilita uma forte recuperação na saída das crises, como ocorreu, por exemplo, em 2010. 
média da economia brasileira está mais próxima dos 16\% do que dos 17\%.

\subsection{TAXA DE CRESCIMENTO DAS HORAS TRABALHADAS}

A série de horas trabalhadas utilizada neste estudo é uma junção das horas trabalhadas da PNAD e da PME. Para os anos de 1995 a 2001 utiliza-se os dados da PNAD. Para os anos de 2002 a 2010, utiliza-se a série de horas construída por Barbosa Filho e Pessôa(2009) ${ }^{4}$.

A Tabela 4 mostra a taxa de crescimento da mão de obra por diversos períodos. A taxa de crescimento das horas trabalhadas foi próxima aos 2,4\% ao ano no período 2004 -2008. A mesma desacelerou no período 2007-2010, para 1,8\% ao ano. Entre 2009 e 2011, a taxa de crescimento das horas trabalhadas caiu para 1,6\% ao ano. Desta forma, pode-se constatar que o crescimento das horas trabalhadas tem contribuído de forma importante com o crescimento do produto potencial, mas reduzindo-se nos últimos anos.

TABELA 4 - TAXA DE CRESCIMENTO DAS HORAS TRABALHADAS

\begin{tabular}{ccc}
\hline \multicolumn{3}{c}{ Média por período } \\
\hline $1995-2010$ & 3253238725 & $2,0 \%$ \\
$2003-2010$ & 3560769766 & $2,3 \%$ \\
$2007-2010$ & 3698226011 & $1,8 \%$ \\
$2003-2008$ & 3500954320 & $2,6 \%$ \\
$2004-2008$ & 3545717043 & $2,4 \%$ \\
$2009-2011$ & 3784193038 & $1,6 \%$ \\
\hline
\end{tabular}

FONTE: Elaboração própria.

\subsection{TAXA DE CRESCIMENTO DO CAPITAL HUMANO}

O processo de universalização da educação no Brasil está em ritmo bastante avançado, com uma taxa de matrícula líquida superior aos $90 \%$ na faixa etária com idade entre 7 e 14 anos de idade, mas com uma taxa líquida de matrícula pouco superior aos 50\% para os jovens com idades entre 15 e 17 anos. As duas taxas de matrícula continuam se elevando ao longo do tempo, representando o investimento em educação. Entretanto, em virtude de o capital humano ser uma variável com grande inércia e resultado da soma de investimentos realizados no passado, o investimento atual em educação provoca elevações suaves no capital humano. A Tabela 5, de Barbosa Filho (2011), mostra a taxa de crescimento anual média do capital humano no Brasil.

TABELA 5 - ESTOQUE DE CAPITAL HUMANO (VARIAÇÃO MÉDIA POR PERÍODO)

\begin{tabular}{c|r|r|r}
\hline Período & Anos de escolaridade & BK & BPV \\
\hline $1995-2010$ & $2,4 \%$ & $1,7 \%$ & $1,3 \%$ \\
$2003-2010$ & $2,0 \%$ & $1,5 \%$ & $0,9 \%$ \\
$2007-2010$ & $1,8 \%$ & $1,3 \%$ & $0,8 \%$ \\
$2003-2008$ & $2,2 \%$ & $1,6 \%$ & $1,2 \%$ \\
$2004-2008$ & $2,0 \%$ & $1,4 \%$ & $1,0 \%$ \\
\hline
\end{tabular}

4 Os autores utilizam o nível da série da Pesquisa Nacional por Amostra de Domicílios (PNAD) e incorporam os movimentos mensais da Pesquisa Mensal de Empregos (PME), ambas do IBGE. 
FONTE: Barbosa Filho (2011).

A Tabela 5 mostra que a taxa de crescimento do capital humano do país ocorre a um ritmo menor nas duas mensurações de capital humano do que o crescimento médio dos anos de escolaridade. O crescimento aparentemente baixo dos anos de escolaridade decorre da inércia desta variável.

No período 2007-2010, o capital humano cresceu em média 1,3\% mensurado à la BK e $0,8 \%$ à la BPV.

\subsection{TAXA DE CRESCIMENTO DO PRODUTO POTENCIAL}

Para calcular a taxa de crescimento do produto potencial, utilizamos a taxa de crescimento da PTF, do capital, da mão de obra e do capital humano para cada um dos períodos analisados (como registrados nas Tabelas 2, 3, 4 e 5, respectivamente) na Equação (4).

Os resultados mostram que o produto potencial da economia brasileira nos períodos analisados atingiu um máximo de 4,4\% no período 2004 -2008 com capital humano à la BPV e um máximo de 4,3\% entre 2007 e 2010 com capital humano à la BK. Desta forma, com base nas variações passadas dos fatores de produção: capital, horas trabalhadas, capital humano e PTF, pode-se afirmar que na melhor das hipóteses a taxa de crescimento do produto potencial foi de $4,5 \%$ nos últimos anos.

TABELA 6 - CRESCIMENTO DO PRODUTO POTENCIAL

\begin{tabular}{c|r|r}
\hline \multirow{2}{*}{ Período } & \multicolumn{2}{|c}{ Capital Humano $a ̀ l a$} \\
\cline { 2 - 3 } & \multicolumn{1}{|c|}{ BK } & \multicolumn{1}{c}{ BPV } \\
\hline $1995-2010$ & $2,9 \%$ & $2,2 \%$ \\
$2003-2010$ & $3,6 \%$ & $3,7 \%$ \\
$2007-2010$ & $4,3 \%$ & $4,3 \%$ \\
$2003-2008$ & $3,7 \%$ & $3,7 \%$ \\
$2004-2008$ & $4,2 \%$ & $4,4 \%$ \\
\hline
\end{tabular}

FONTE: Elaboração própria.

Apesar de ilustrativos, os cálculos apresentados na Tabela 6 apresentam uma taxa de crescimento do produto potencial relacionada ao passado. Para que possamos analisar o que ocorrerá com o produto potencial no futuro devemos realizar projeções acerca da taxa de crescimento das horas trabalhadas, do capital, do capital humano e da PTF.

\section{PRODUTO POTENCIAL FUTURO}

A projeção da variação do total de horas trabalhadas é realizada com base na variação do total de horas dos últimos anos. A variação do estoque de capital humano futura é uma 
variável relacionada com o ritmo de expansão dos anos médios de escolaridade da PEA e está baseada nas variações observadas na Tabela 5 .

Para a variação do capital físico e da PTF, utilizam-se diversos cenários com taxas de investimento e variação da PTF distintas.

\subsection{CRESCIMENTO DAS HORAS TRABALHADAS}

A variação no total de horas trabalhadas entre 2001 e 2009 foi em média de 2,1\% ao ano. Entretanto, como pode ser visto na Tabela 4, entre 2009 e 2011 as horas totais trabalhadas aumentaram somente $1,6 \%$ ao ano.

Dada a queda na taxa de desemprego ocorrida nos períodos recentes ${ }^{5}$, é difícil de imaginar que uma nova redução na taxa de desemprego possa contribuir ainda mais para a elevação do total de horas trabalhadas em nível tão acelerado (2,1\%). Desta forma, as horas trabalhadas devem crescer a uma taxa de 1,5\% ao ano, próxima a taxa média de 1,6\% observada nos últimos três anos. Um crescimento de 1,5\% ao ano do total de horas trabalhadas significa uma contribuição de $0,9 \%$ ao ano de crescimento no produto potencial.

\subsection{CRESCIMENTO DO CAPITAL HUMANO}

A taxa de crescimento do capital humano deve continuar a contribuir de forma positiva com o crescimento do produto potencial nos próximos anos. Como mostra a Tabela 5, os anos médios de escolaridade crescem no Brasil entre 0,1 e 0,15 anos de estudo ao ano. Este processo deve continuar nos próximos anos visto que as taxas de matrícula no país continuam a subir no ensino médio e que a educação média ainda é bastante baixa (7,6 anos segundo a PNAD de 2009).

Neste ritmo, para que a economia possa atingir 11 anos médios de escolaridade serão necessários pelo menos 20 anos de elevação dos anos médios de escolaridade na faixa de $2 \%$ ao ano, o que acarretaria em uma elevação do capital humano em torno de $1 \%$ ao ano ${ }^{6}$. Esta taxa de crescimento do capital humano contribuiria em torno de $0,6 \%$ ao ano para o crescimento do PIB.

\subsection{CRESCIMENTO DO CAPITAL}

A taxa de crescimento do capital físico na economia brasileira foi relativamente baixa

5 A taxa de desemprego medida pela PME em setembro de 2003 estava em 13\%, em setembro 2009 estava em $7,7 \%$, enquanto a mesma reduziu-se para 6,2\% em setembro de 2010 e 6,0\% em setembro de 2011.

6 Taxa de crescimento do capital humano intermediária a observada no período 2007-2010 para as duas séries de capital humano utilizadas. 
nos últimos anos, sendo o tendão de Aquiles da economia brasileira. Com base na Equação (5), que mostra a importância da taxa de investimento $\left(\frac{I}{Y}\right)$ para a elevação do estoque de capital, percebe-se que a taxa de investimento em valores constantes da economia brasileira situa-se em nível bastante baixo (Tabela 3). Para que a taxa de crescimento do capital $(\dot{K} / K)$ possa contribuir de forma mais vigorosa para o crescimento do produto potencial $\left(\frac{\dot{Y}}{Y}\right)$ no país necessitamos de uma elevação substancial da taxa de investimento $\left(\frac{I}{Y}\right)$. A Tabela 7 mostra a relação entre elevação do investimento, do capital e do produto, dada uma relação capital produto $\left(\frac{K}{Y}\right)$ de 2,54 e uma taxa de depreciação de $3,5 \%$ ao ano.

TABELA 7 - IMPACTO DA TAXA DE INVESTIMENTO SOBRE O ESTOQUE DE CAPITAL E O PRODUTO

FONTE: Elaboração própria.

\begin{tabular}{r|c|c}
\hline$\left(\frac{I}{Y}\right)$ & $\left(\frac{\dot{K}}{K}\right)$ & $\left(\frac{\dot{Y}}{Y}\right)$ \\
\hline $16 \%$ & $2,8 \%$ & $1,1 \%$ \\
$18 \%$ & $3,6 \%$ & $1,4 \%$ \\
$20 \%$ & $4,4 \%$ & $1,8 \%$ \\
$22 \%$ & $5,2 \%$ & $2,1 \%$ \\
$24 \%$ & $6,0 \%$ & $2,4 \%$ \\
$26 \%$ & $6,8 \%$ & $2,7 \%$ \\
\hline
\end{tabular}

Com base na Tabela 7 podemos perceber que para elevar a taxa de crescimento do produto potencial por volta de $1 \%$ temos que elevar a taxa de investimento em torno de $6 \%$, um esforço importante. Entretanto, para que esta elevação da taxa de investimento seja possível o país necessita elevar a sua taxa de poupança (na faixa dos 18\%), algo difícil de acreditar com a atual política fiscal em curso.

\subsection{PRODUTIVIDADE TOTAL DOS FATORES}

A Tabela 2 mostra como a PTF no país é uma variável volátil. Ao mesmo tempo, um maior crescimento da PTF é fundamental para alavancar a taxa de crescimento do produto potencial.

Desta forma, para prever a capacidade de crescimento do produto potencial da economia brasileira vamos admitir diversos valores para a taxa de crescimento da PTF. Consideramos um crescimento da PTF de 1,5\% ao ano, uma taxa bastante otimista.

\subsection{PRODUTO POTENCIAL}

Com base nas previsões de expansão das horas trabalhadas de 1,5\% ao ano, de $1 \%$ ao ano do capital humano e cinco cenários distintos de crescimento da PTF e da taxa de investi- 
mento, calcula-se o produto potencial da economia brasileira.

A Tabela 8 mostra os resultados do cálculo do produto potencial da economia brasileira com base nas premissas acima e com diferentes PTFs e taxas de investimento.

TABELA 8 - PRODUTO POTENCIAL

\begin{tabular}{l|r|r|r|r|r}
\hline \multirow{2}{*}{$\begin{array}{c}\text { Crescimento } \\
\text { PTF }\end{array}$} & \multicolumn{5}{|c}{ Taxa de investimento } \\
\cline { 2 - 7 } & $\mathbf{1 6 , 0 \%}$ & $\mathbf{1 7 , 0} \%$ & $\mathbf{1 8 , 0} \%$ & $\mathbf{1 9 , 0} \%$ & $\mathbf{2 0 , 0} \%$ \\
\hline $0,5 \%$ & $3,1 \%$ & $3,3 \%$ & $3,4 \%$ & $3,6 \%$ & $3,8 \%$ \\
$1,0 \%$ & $3,6 \%$ & $3,8 \%$ & $3,9 \%$ & $4,1 \%$ & $4,3 \%$ \\
$1,5 \%$ & $4,1 \%$ & $4,3 \%$ & $4,4 \%$ & $4,6 \%$ & $4,8 \%$ \\
$2,0 \%$ & $4,6 \%$ & $4,8 \%$ & $4,9 \%$ & $5,1 \%$ & $5,3 \%$ \\
$2,5 \%$ & $5,1 \%$ & $5,3 \%$ & $5,4 \%$ & $5,6 \%$ & $5,8 \%$ \\
\hline
\end{tabular}

FONTE: Elaboração própria.

Com base nos resultados apresentados na Tabela 8, pode-se observar que para o produto potencial crescer a uma taxa próxima aos $4,5 \%$ ao ano é necessário realizar um investimento de $18 \%$ e ter um crescimento da PTF de 1,5\% ao ano. Este crescimento parece um tanto elevado, dado o crescimento estimado da PTF de 0,6\% em $2011^{7}$.

Apesar da elevada volatilidade da PTF, a baixa estimativa da mesma para 2011, associada com a baixa expectativa de crescimento para 2012 sugerem que a taxa de crescimento da PTF nos próximos anos deve ficar mais próxima de 1\% do que de 1,5\%. Isto significa que o produto potencial brasileiro com uma taxa de investimento a preços constantes de $17 \%$ está próximo dos 3,8\% ao ano e não dos 4,3\% ao ano.

A recente queda da PTF pode ser explicada por diversos fatores. Neste artigo levantamos duas hipóteses para a redução da PTF nos últimos anos. Na primeira hipótese, a crise subprime de 2008 pode ter causado efeitos permanentes sobre a produtividade da economia, uma vez que a indústria parece ter sido o setor mais atingido pela crise. Como a recuperação da economia deslocou a mesma em direção de setores de produtividade mais baixa, é possível que a PTF global da economia caia.

Outra possibilidade é que as intervenções realizadas nos últimos governos deterioram as instituições do país (perda de importância das agências reguladoras, por exemplo) e que a intervenção estatal mais elevada nos últimos anos (papel bastante ativo do BNDES, por exemplo) tenham reduzido a eficiência econômica no país, acarretando em uma redução da PTF.

Estes temas devem ser analisados no futuro, uma vez que para elevar a taxa de crescimento do produto potencial, um aumento da PTF é fator crucial.

7 Assumindo um crescimento do produto real de 3,5\% no ano, um crescimento de 1\% ao do capital humano e utilizando o crescimento observado do estoque de capital de $3,8 \%$ e das horas trabalhadas de $1,9 \%$. 


\section{CONCLUSÃO}

Este artigo calculou a taxa de crescimento do produto potencial da economia brasileira. Os resultados sugerem que esta taxa encontrava-se próxima dos $4,4 \%$ ao ano no período 2007-2010. O crescimento do total de horas trabalhadas deve diminuir em torno de $0,5 \%$ ao ano em um futuro próximo, pois não parece haver mais espaço para grandes reduções da taxa de desemprego. Logo, a contribuição das horas trabalhadas para o crescimento do produto potencial deve ser reduzida em $0,3 \%$ ao ano.

A contribuição do capital humano para o crescimento do produto potencial deve continuar em torno de $0,6 \%$ ao ano nas próximas décadas, sendo um importante fator para o crescimento do país. A taxa de investimento realizado no Brasil é um entrave a um crescimento mais elevado. Neste caso, a baixa taxa de poupança é um limitador do mesmo.

A taxa de crescimento futura do produto potencial de 4,3\% ao ano somente é compatível com uma taxa de investimento de $17 \%$ ao ano e um crescimento da PTF de 1,5\% ao ano, ou com um investimento de $20 \%$ e um crescimento da PTF de 1\%. Dado que a PTF estimada para o ano de 2011 apresenta um crescimento de 0,6\%, é bastante plausível que o produto potencial da economia esteja pelo menos 0,5\% abaixo do registrado no período 2007-2010, com um crescimento abaixo dos 3,8\% do PIB.

\section{REFERÊNCIAS}

BARBOSA FILHO, F. PTF, horas trabalhadas e produto potencial no Brasil, 2011. Mimeo.

BARBOSA FILHO, F.; PESSÔA, S. de A.; VELOSO, F. A. Evolução da produtividade total dos fatores na economia brasileira com ênfase no capital humano - 1992-2007. Revista Brasileira de Economia, Rio de Janeiro, v. 64, n. 2, p. 91-113, 2010.

BARBOSA FILHO, F.; PESSÔA, S. de A. Série de horas mensais da economia brasileira, 2009. Mimeo.

BILS, M.; KLENOW, P. J. Does schooling cause growth? American Economic Review, v. 90 n. 5, p. 1160-1183, 2000.

GOMES, V.; PESSÔA, S. de A.; VELOSO, F. A. Evolução da produtividade total dos fatores na economia brasileira: uma análise comparativa. Pesquisa e Planejamento Econômico, v. 33, n. 3, p. 389-434, 2003.

MINCER, J. Schooling, experience and earnings. Nova York: Columbia University Press, 1974. 